\title{
SUBSEROSAL HAEMATOMA OF THE ILEUM
}

\author{
BY \\ ANTONIO GENTIL MARTINS \\ From the Department of Surgery, Alder Hey Children's Hospital, Liverpool
}

(RECEIVED FCR PUBLICATION DECEMBER 21, 1959)

Angiomas of the ileum are rare. Their association with a duplication cyst has not so far been described. The unusual mode of presentation, with intestinal obstruction and a palpable mass (subserosal haematoma) simulating intussusception, have prompted the report of the present case.

\section{Case Report}

N.C., a white male infant, born June 18 , 1958, was admitted to hospital on May 18, 1959, when 11 months old, with a five days' history of being irritable and apparently suffering from severe colicky abdominal pain for the previous 24 hours. On the day of admission his bowels had not moved and he vomited several times.

He looked pale and ill and a mass could be felt in the right hypochondrium. There was no significant abdominal distension and the bowel sounds were within normal limits. A saline enema was given which showed a small amount of mucus but no blood. A diagnosis of intussusception was made, and after resuscitation with intravenous therapy the same evening laparotomy was performed, which showed no evidence of intussusception, but instead a large subserosal haematoma, involving approximately $7 \mathrm{~cm}$. of ileum, $30 \mathrm{~cm}$. from the ileo-caecal junction, and which formed the tumour that had been felt clinically (Figs. 1 and 2). In the middle of the haematoma there was a hard yellow small tumour; the mesenteric glands were slightly enlarged and some were blueish in colour. Resection of the diseased segment with end-to-end anastomosis was performed, and the appendix was removed.

The child remained ill for a few days but recovered and was discharged home on the seventh postoperative day, when he was taking feeds well and gaining weight.

When last seen, two months after operation, he was thriving and no further appointment was considered necessary.

The appendix, as expected, showed no macro- or microscopic abnormality; the mesenteric gland showed, on microscopic examination, only sinus catarrh. The resected gut, on macroscopic examination, showed 'a segment of intestine $9 \mathrm{~cm}$. long, half way along which there was a haemorrhage over an area of $4 \mathrm{~cm}$., which had lifted the serosa from the rest of the wall. On the outer aspect, also half way, there was a small diverticulum $1 \mathrm{~cm}$. long, which, however, did not appear to communicate with the lumen of the small bowel. Opposite, the mucosa had a small erosion'.

Microscopical examination (Figs. 3, 4 and 5) showed that "considerable haemorrhage had occurred in the serous, muscular and mucous coats. The mucosa, however, was viable and the maximal zone of damage was towards the serosa. Numerous large capillaries were present in the coats. The lining of the diverticulum formed by glandular epithelium suggesting ileal mucosa was partly destroyed, but it had a well-formed muscular coat': it was considered to be probably a duplication. The main diagnosis was that of haemangioma of the ileum.

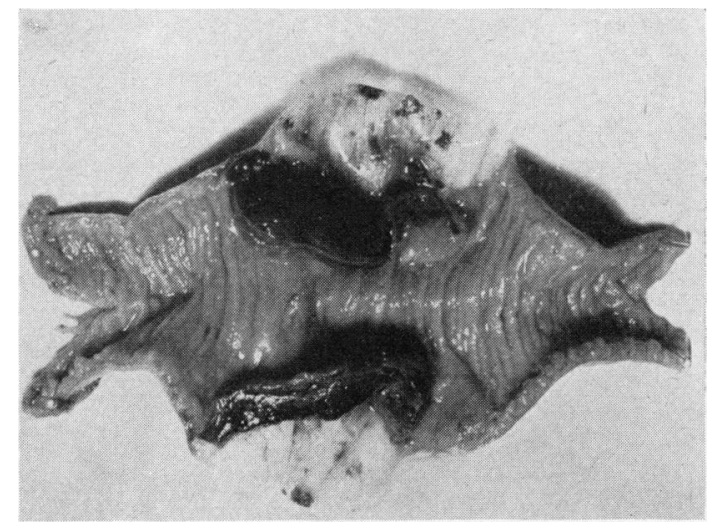

FIG. 1.-Resected specimen showing subserosal haematoma and narrowing of the intestinal lumen as well as a small mucosal erosion at the level of the duplication.

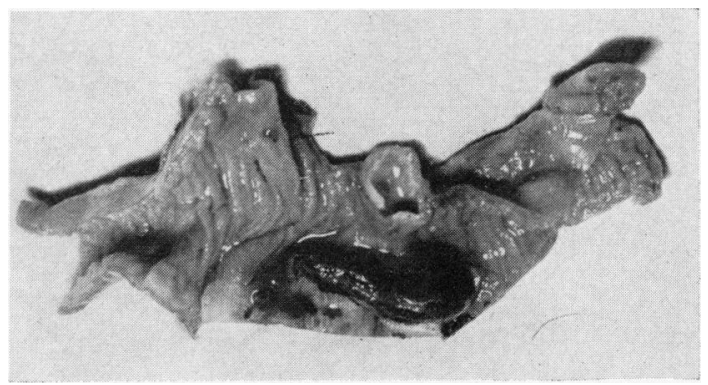

Fig. 2.-Resected specimen showing duplication cyst. 


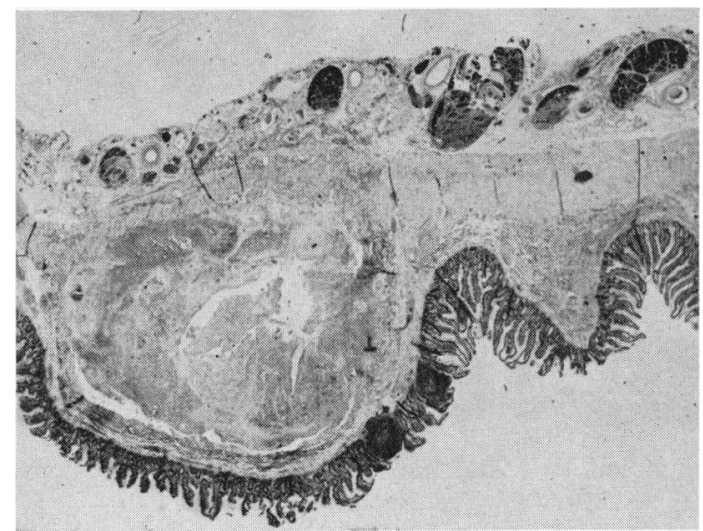

FIG. 3.-Low power magnification showing numerous vascular spaces in the subserosal area and a large area of haemorrhage in the submucosa.

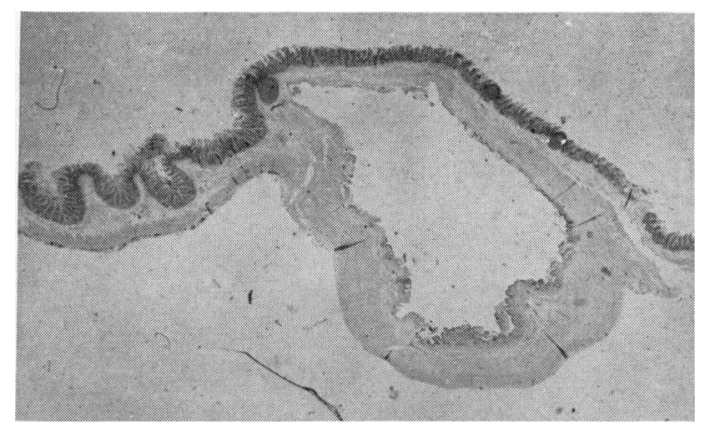

FIG. 4.-Low power magnification of the duplication cyst and the common muscular wall with the intestine.

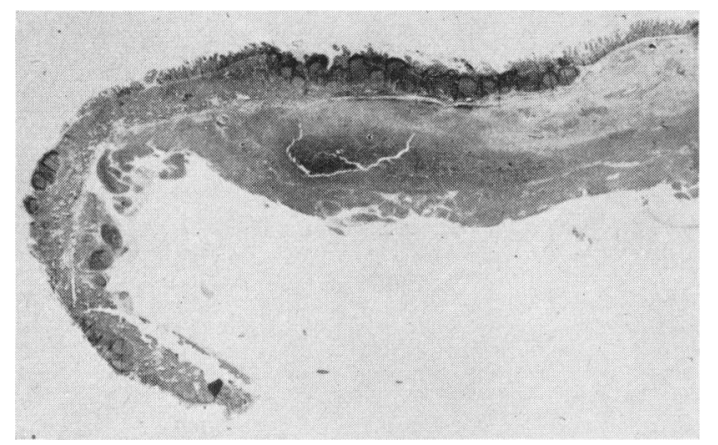

Fig. 5.-Lower power magnification in the main zone of haematoma formation, with haemorrhagic infiltration of all the bowel wall.

\section{Discussion}

As haemangiomas are common in children it is surprising to find how rarely they involve the gastrointestinal tract. The great majority of cases have been reported in adult life. According to Hansen
(1948) only 66 haemangiomas of the small bowel have been reported in the literature, and of these, only two were malignant (Ewing, 1940). Raiford (1932) quoted by Rickham (1952) found, in a series of 11,500 autopsies, that haemangiomas of the small bowel constituted $0 \cdot 3 \%$ of all tumours of the gastrointestinal tract.

They can be diffuse infiltrating lesions with resultant thickening of the wall and narrowing of the lumen, or localized tumours projecting into the lumen. They are sometimes multiple and may be associated with haemangiomas elsewhere.

Simple capillary and/or cavernous haemangioma of the small bowel present frequently with gastrointestinal bleeding and occasionally are the leading point of an intussusception. More rarely they can lead to intestinal obstruction, due to the size of the tumour itself.

We think the case reported here is the first case described which presented by a subserosal haematoma. The differentiation from an intussusception was obviously impossible clinically. Treatment, however, was not influenced by the diagnosis as laparotomy was indicated in both cases.

Subserosal haematomas leading to intestinal obstruction have been reported by Zabin (1952), Lampert, Goodfellow and Wachowski (1954), and Robbarts (1957), but in those cases the children were older, with a definite history of non-penetrating abdominal injury, and examination of the resected specimens did not show haemangiomas. They were usually found near the more fixed zones of the gut, mainly near the duodenojejunal flexure.

The finding of a tumour in the centre of the area of haematoma made us look for some relationship between them, but without success. The tumour was small, smooth, rounded and yellowish, with a well-defined muscular wall in part common to the gut wall. It was cystic, with no communication with the intestinal lumen, and must be considered as a small duplication cyst. Although it is true to say that no agreed theory has so far been presented to explain the formation of those intestinal duplications, it seems certain that embryologically the haemangioma and the duplication have different origins and must be considered as coincidental but unrelated findings. Duplications of the gut, although not frequent, are a well known entity to the paediatric surgeon: Gross (1953) describes 71 cases, of which 19 were in the ileum. In the Alder Hey series Basu (1959) found seven in the ileum, out of a total of 29.

Although intramural haemorrhage in association with duplication of the intestine has been described 
we could not find, in our case, evidence of the bleeding being due to the duplication.

The treatment of subserosal haematoma is resection, particularly when associated with any other bowel lesion, e.g. tumour, duplication. In the traumatic cases also we favour resection and feel that the simple evacuation of the blood clot, although successfully used by Lampert et al. (1954) and Robbarts (1957) should only be employed in exceptional circumstances when resection is too risky or contra-indicated.

\section{Summary}

A case of haemangioma of the ileum in an 11-month-old boy, presenting with subserosal haematoma leading to marked narrowing of the lumen and to intestinal obstruction, and showing a clinical picture typical of intussusception, is reported.
A small duplication of the ileum was found at the same level and considered to be a coincidental but unrelated finding.

My thanks are due to Miss Isabella Forshall for allowing me to publish this case; to Drs. Edward G. Hall and Jean Bouton for the pathological report, and to Mr. Charles Fitzsimons for the microphotographs.

\section{REFERENCES}

Basu, R. (1959). Personal communication.

Ewing, J. (1940). Neoplastic Diseases, 4th ed., p. $249 . \quad$ Saunders, Philadelphia

Gross, R. E. (1953). The Surgery of Infancy and Childhood, p. 168. Saunders, Philadelphia.

Hansen, P. S. (1948). Hemangioma of the small intestine (with special reference to intussusception). Amer. J. clin. Path., $18,14$.

Lampert, E. G., Goodfellow, J. G. and Wachowski, T. J. (1954) Traumatic subserosal hemorrhage causing small bowel obstruction. Ann. Surg., 140, 768.

Raiford, T. S. (1932). Tumors of the small intestine-rare tumorsenterocysts. Arch. Surg. (Chicago), 25, 122, 321.

Rickham, P. P. (1952). A case of haemangiomatosis of the small intestine. Brit. J. Surg., 39, 462.

Robbarts, F. H. (1957). Traumatic intramural haematoma of the proximal jejunal loop. Arch. Dis. Childh., 32, 484.

Zabin, A. (1952). Acute intramural jejunal hemorrhage (case report). N.Y. St. J. Med., 52, 93. 